Revista Destaques Acadêmicos, Lajeado, v. 10, n. 4, 2018. ISSN 2176-3070

DOI: http://dx.doi.org/10.22410/issn.2176-3070.v10i4a2018.2046

http://www.univates.br/revistas

\title{
DETERMINAÇÃO DA COMPOSIÇÃO CENTESIMAL DE CHOCOLATE MEIO AMARGO
}

\author{
Jamile Helena Marques ${ }^{1}$
}

Resumo: O chocolate é um alimento consumido em grande escala, pelo prazer decorrente de sua ingestão. Por sua composição nutricional e alto consumo, associouse o mesmo a ocorrência de doenças. Com isso, expandiu-se o mercado de variações de chocolate, levando a um aumento do consumo de chocolate meio-amargo, por ser considerado menos prejudicial. Este artigo aborda a determinação da composição centesimal do chocolate meio amargo, para fins de comparação com valores descritos na literatura e com a embalagem do produto. Observou-se teores de umidade, cinzas e lipídeos de acordo com os limites da legislação vigente, o que condiciona o produto para o consumo. Assim, o chocolate meio amargo possui valor nutricional que permite seu consumo de forma consciente, apresentando benefícios. A amostra apresentou umidade de $0,81 \%$, teor de cinzas de $1,59 \%$, teor de lipídeos de 30,33\%, teor de fibras de $13,43 \%$, teor de proteínas de $5,73 \%$ e teor de carboidratos de $48,08 \%$. Além disso, a amostra analisada apresentou valor calórico de 486,47 kcal/100 g, sendo que em comparações com resultados da literatura todas possuem alto valor calórico e teor de carboidratos, o que configura o chocolate com um valor com alto teor energético.

Palavras-chave: Chocolate Meio Amargo; Composição Centesimal; Benefícios.

\section{INTRODUÇÃO}

O chocolate é um produto amplamente consumido em todo o mundo, muito mais por prazer do que por nutrição, e acredita-se que por isso tenha alcançado extremo sucesso entre os consumidores (BOLENZ et al., 2003). Cohen et al. (2004) afirmam que o Brasil é o quinto maior produtor de chocolate do mundo, sendo, devido a isso, o elevado consumo do produto no país, sendo também o terceiro maior produtor.

O chocolate é definido como sendo uma suspensão de partículas sólidas não-gordurosas, como açúcar e sólidos de cacau, em uma fase gordurosa contínua de manteiga de cacau (CHEVALLEY, 1994).

1 Acadêmica do Curso de Farmácia da Universidade de Santa Cruz do Sul (UNISC). jamilehmarques@gmail.com 
Segundo a ANVISA (Agência Nacional de Vigilância Sanitária), chocolate é o produto preparado com cacau obtido por processo tecnológico adequado, e açúcar, podendo conter ouras substâncias aprovadas. O chocolate meio amargo é preparado com maior proporção de pasta e manteiga de cacau, com pouca quantidade de açúcar (BRASIL, 2005).

Os europeus, no século XVI, utilizavam o cacau e chocolate (líquido) como veículos de medicamentos, sendo considerados por si só como medicamentos. $\mathrm{Na}$ forma isolada ou em combinação com ervas, plantas e outros suplementos alimentares, ambos eram utilizados no tratamento de doenças, como desordens digestivas, dores de cabeça, inflamações e insônias (KWIKURIBE, 2005).

Alguns anos após, o chocolate deixou de ser considerado um alimento terapêutico, em decorrência de seu consumo excessivo e composição nutricional, o que estiveram associados a diversas patologias (LIPPI et al., 2009).

Assim, quando consumido de maneira consciente, o mesmo possui diversas propriedades benéficas para a saúde, devido ao fato de seus componentes apresentarem diversas propriedades que auxiliam no tratamento e prevenção de diversas doenças (PIMENTEL, 2007; OLIVEIRA et al., 2009; JEZUS, 2010).

Atualmente há um aumento no consumo de chocolate meio amargo, devido as suas características funcionais, pela presença de substâncias bioativas, por sua capacidade antioxidante, que reduz a incidência de patologias crônicodegenerativas, principalmente as doenças cardiovasculares, pela presença de flavonoides. (SOUZA, 2010).

O chocolate, contendo quantidade elevada de polifenóis, contribui para potente atividade antioxidante e proteção de dados ao DNA das células, além de formação de radicais livres (WOLLGAST; ANKLAN, 2000).

Diversos estudos foram realizados nos últimos anos para criar diversos tipos e sabores de chocolates, tendo em vista a apreciação e buscando atender as necessidades dos consumidores, que anseiam por produtos diferenciados, com menores teores de gorduras, visando equilibrar o consumo do chocolate e uma alimentação saudável (RICHTER; LANNES, 2007).

Devido a isso, a conhecimento da composição e do valor nutricional dos alimentos dá-se como base para uma educação nutricional, servindo para a adequação alimentar da população (BORGES et al., 2001).

No Brasil, dados de produção de chocolate demonstram que, em 2011, a produção foi de 710 mil toneladas. De acordo com a Associação Brasileira da Indústria de Chocolates, Cacau, Amendoim, Balas e derivados (ABICAB), o Brasil é o terceiro maior produtor de chocolates do mundo e quarto em consumo, pois o brasileiro ingere cerca de $2,2 \mathrm{~kg}$ de chocolate por ano (ABICAB, 2012). 
A partir disto, realizou-se o presente estudo, com o objetivo de determinar a composição centesimal do chocolate meio amargo, por seu consumo estar sendo difundido no país.

\section{METODOLOGIA}

Para a realização do presente estudo, foi analisada uma amostra de chocolate meio amargo, adquirida em um supermercado de Santa Cruz do Sul - RS. A partir disto, foi realizada a determinação da composição centesimal da amostra, tendo sido realizadas análises de umidade, cinzas, proteínas, fibras e gorduras totais, em triplicata. As análises dos teores de cinzas e umidade foram realizadas conforme preconizado pelo Instituto Adolfo Lutz, de 2008. A determinação do teor de fibras foi feita pelo método gravimétrico de Hannenberg, sugerido pelo Instituto Adolfo Lutz, de 2008. As análises para determinação de proteínas e lipídeos foram realizadas conforme AOAC, de 2011.

O teor de carboidratos foi obtido por diferença. O cálculo do valor calórico foi realizado utilizando os fatores de conversão determinados na RDC $\mathrm{n}^{\circ} 40$ (HOLLAND, et al., 1994).

Foi feita a determinação da umidade através da secagem do material sob aquecimento em estufa a $105^{\circ} \mathrm{C}$ por 3 horas até obtenção de peso constante (IAL, 2008). O teor de cinzas foi obtido através de incineração do material em mufla a $550^{\circ} \mathrm{C}$ até obtenção de cinzas branco-acinzentadas e peso constante (IAL, 2008).

A determinação de proteínas foi feita por meio do teor de nitrogênio total, pelo método Kjeldahl, determinado ao nível semimicro (AOAC, 2011), com fator de conversão do nitrogênio para proteína de 6,25. As fibras foram determinadas através do método de digestão ácida seguida de digestão básica (IAL, 2008).

A determinação de lipídios totais foi feita através do método por extração em aparelho Soxhlet com utilização de éter de petróleo como solvente (AOAC, 2011). Os carboidratos totais foram calculados pela diferença: al., 1994).

100 - (\% umidade $+\%$ cinzas $+\%$ proteína $+\%$ lipídios) (HOLLAND et

O valor calórico da amostra foi calculado pela soma das porcentagens de proteína e carboidratos totais multiplicados pelo fator 4 somado ao teor de lipídios totais multiplicados pelo fator 9 (HOLLAND et al., 1994).

\section{RESULTADOS E DISCUSSÃO}

A partir das análises realizadas, foram obtidos resultados, os quais estão apresentados na Tabela 1. Os valores obtidos relacionados a amostra A, referemse as análises realizadas no laboratório de bromatologia da Universidade de 
Santa Cruz do Sul - RS. As análises foram realizadas uma única vez. Para fins de comparação, foram utilizadas as informações descritas na embalagem do produto, a legislação vigente e a Tabela Brasileira de Composição de alimentos - TACO (UNICAMP, 2011). A amostra A corresponde a amostra analisada, em base seca, enquanto a amostra B corresponde aos valores descritos por VISSOTTO et al. (1999).

Tabela 1 - Composição centesimal do chocolate meio amargo

\begin{tabular}{l|c|c|c|c|c|c}
\hline \multicolumn{1}{c|}{ Ensaio } & Amostra A & Amostra B & Amostra C & Amostra D & Legislação & $\begin{array}{c}\text { Tabela } \\
\text { TACO }\end{array}$ \\
\hline Umidade (\%) & $0,81 \pm 0,08$ & 0,8 & 1,0 & 0,56 & Máx. 3\% & - \\
\hline Cinzas (\%) & $1,59 \pm 0,14$ & 0,9 & 2,4 & 1,87 & Máx. 2,5\% & - \\
\hline Lipídeos (\%) & $30,33 \pm 0,34$ & 31,5 & 34,3 & - & Mín. 30\% & - \\
\hline Proteínas (\%) & $5,73 \pm 0,12$ & 4,2 & 14,4 & - & - & 4,9 \\
\hline Fibras (\%) & $13,43 \pm 0,08$ & - & - & - & - & 4,9 \\
\hline Carboidratos (\%) & $48,08 \pm 0,25$ & 62,6 & 47,8 & - & - & 62,4 \\
\hline $\begin{array}{l}\text { Valor calórico } \\
\text { (kcal/100 g) }\end{array}$ & $486,47 \pm 0,05$ & 550,0 & 557,5 & - & - & 475 \\
\hline
\end{tabular}

A partir dos resultados obtidos por meio das análises realizadas, foi feita a comparação com resultados descritos na literatura.

Em relação a umidade, a amostra A apresentou 0,81\% de umidade, enquanto a amostra $B$ apresentou $0,8 \%$ de umidade, sendo ambos valores iguais. Conforme a legislação vigente, ambos estão de acordo com o preconizado, que é de, no máximo, 3\% de umidade (BRASIL, 1978).

$\mathrm{Na}$ análise do teor de cinzas, a amostra A apresentou 1,59\% de cinzas, enquanto a amostra B apresentou $0,9 \%$ de cinzas. Ambas estão de acordo com o preconizado pela legislação, que é de, no máximo 2,5\% de cinzas (BRASIL, 1978).

Em relação ao teor de lipídeos, as análises indicaram 30,33\% de lipídeos na amostra $\mathrm{A}$, enquanto que as análises da amostra B indicam 31,5\% de lipídeos. Ambos são valores simultâneos e que estão de acordo com o valor exigido pela legislação, que indica teor mínimo de lipídeos de 20\% (BRASIL, 1978).

A amostra A apresentou $13,43 \%$ de fibras, enquanto que este teste não foi realizado na amostra B. Porém, na embalagem do chocolate analisado, o fabricante indica que o chocolate contém $10 \%$ de fibras. Ambos são valores próximos, porém discrepantes do que indica a Tabela TACO (UNICAMP, 2011) que indica $4,9 \%$ de fibras. 
O teor de proteínas na amostra A foi de 5,73\%, enquanto a amostra B apresentou 4,2\% de proteínas. Conforme dados da embalagem, o produto contém 5,6\% de fibras, estando o valor encontrado na amostra A muito próximo ao valor descrito pela embalagem, sendo semelhante ao valor descrito pela Tabela TACO, de 4,9\% de proteínas.

Em relação ao teor de carboidratos, a amostra A apresentou $48,08 \%$ de carboidratos, enquanto que a amostra B indica $62,6 \%$ de carboidratos. Conforme a embalagem, o produto deve apresentar $52 \%$ de carboidratos, semelhante ao valor encontrado pelas análises realizadas na amostra A. Porém, a tabela TACO indica $62,4 \%$ de carboidratos, semelhante ao encontrado pela análise realizada na amostra B.

Em relação ao valor calórico, segundo a embalagem, o produto apresenta $500 \mathrm{kcal} / 100 \mathrm{~g}$, enquanto análises na amostra A demonstram valor próximo, de $486,47 \mathrm{kcal} / 100 \mathrm{~g}$. Segundo a tabela TACO o valor energético do produto é de $475 \mathrm{kcal} / 100 \mathrm{~g}$, semelhante ao valor encontrado conforme as análises realizadas.

A Figura 1 representa o esquema gráfico da determinação da composição centesimal do chocolate meio amargo, conforme as análises realizadas.

Figura 1 - Determinação da composição centesimal (Amostra A)

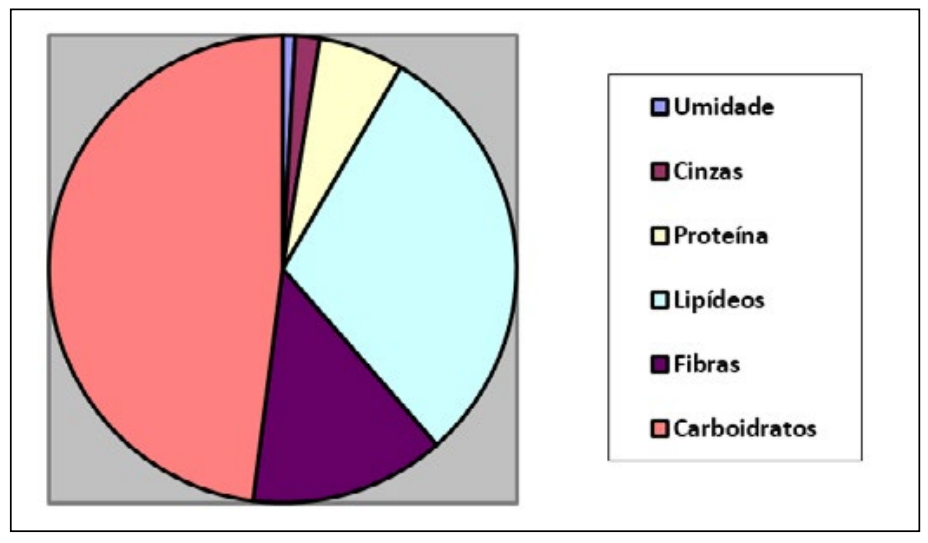

Com base nas análises realizadas e conforme demonstra a Figura 1, pode-se constatar que a amostra A apresenta em sua composição um maior percentual de carboidratos, sendo estes responsáveis por quase metade da composição centesimal da amostra.

Conforme as análises realizadas por VISSOTTO et al. (1999), a amostra B também apresenta grande quantidade de carboidratos, o que equivale a grande parte da composição centesimal do produto.

Conforme análises de LORENZO (2017), a amostra C apresentou valores semelhantes aos encontrados na amostra analisada, com alto teor 
de carboidratos e estando de acordo com os parâmetros preconizados pela legislação. Além disso, a mesma apresentou valor calórico semelhante a amostra analisada e a tabela TACO.

A amostra D, de acordo com os resultados obtidos por FORTES et al. (2016), que analisou teores de umidade e cinzas em amostras de chocolate meio amargo, o mesmo teve como resultados valores semelhantes aos encontradas nas análises realizadas na amostra $\mathrm{A}$, e os mesmos estavam de acordo com o preconizado pela legislação vigente.

\section{CONCLUSÃO}

A partir da realização deste estudo, foi possível determinar a composição centesimal do chocolate meio amargo. Dado o exposto, a partir das análises, da comparação realizada com dados descritos na literatura e conforme a legislação vigente, percebe-se que, de acordo com os valores obtidos nas análises da amostra A, a mesma apresenta teores de umidade, cinzas e lipídeos que estão de acordo com os parâmetros estabelecidos pela legislação vigente. Quanto às demais análises realizadas, não há valores na legislação que estabelecem parâmetros, porém estes estão semelhantes aos valores encontrados na embalagem do produto e de acordo com a Tabela TACO. A amostra analisada possui as características necessárias, com os teores de acordo com previstos em legislação, podendo ser uma alternativa ao consumo de outros tipos de chocolate, como o ao leite, por exemplo.

\section{REFERÊNCIAS}

Associação Brasileira da Indústria de Chocolates, Cacau, Amendoim, Balas e Derivados - ABICAB. Disponível em: http:/ / www.abicab.org.br/. Acesso em: março de 2018 .

AOAC. Official Methods of Analysis of AOAC International. 18. Ed. Gaithersburg: AOAC, 2011..

BRASIL, Leis, Decretos, etc. Resolução número 12/78 da Comissão Nacional de Normas e Padrões para Alimentos. Diário Oficial, Brasília, 24 de julho de 1978. Seção I, parte 1, p. 11499-11527.

BRASIL. Agência Nacional de Vigilância Sanitária. Resolução RDC n. 264, de 22 de setembro de 2005. Aprova o "Regulamento Técnico para Chocolate e Produtos de Cacau". Diário Ofi cial [da] União, Brasília, set. 2005.

BOLENZ, S. THIESSENHUSEN, T. SCHÄPE, R. Fast conching for milk chocolate. Eur Food Res Technol, Berlin, v. 218, p. 62-67, 2003. 
BORGES, João Tomaz Da Silva et al. Caracterização físico-química e sensorial de pão de sal enriquecido com farinha integral de linhaça. Boletim do Centro de Pesquisa de Processamento de Alimentos, v. 29, n. 1, 2011.

CHEVALLEY, J. Chocolate Flow Properties. In: Industrial Chocolate Manufacture and Use. Editado por S.T. Beckett, 2. ed. England:Clays Ltd., p.139-155, 1994.

COHEN, K.O. LUCCAS, V. JACKIX, M.N.H. Review: Tempering of precrystallization of chocolate. Brasilian Journal of Food Technology, Campinas, v.7, n.1, p.23-29, 2004.

FORTES, M.M., MACEDO, B.L.C., LEAL, J.C., SILVA, P.D.F., CARVALHO, L.M.F., ABREU, J.M. Análise Química do Chocolate Meio Amargo, diet e sem lactose. XXV Congresso Brasileiro de Ciência e Tecnologia de Alimentos. Gramado, 2016.

HOLLAND, B., WELCH, A.A., UNWIN, I.D., BUSS, D.H., PAUL, A.A., SOUTHGATE. MacCance and Winddoeson.s. The composition of foods. $5^{\circ}$ ed., The royal society of chemistry and ministry of agriculture, fisheries and food. Cambridge, U.K., 1994, $462 \mathrm{p}$.

IAL. INSTITUTO ADOLFO LUTZ. Normas Analíticas do Instituto Adolfo Lutz: métodos físico-químicos para análises de alimentos. São Paulo: IAL, 2008.

JEZUS, G. R. Chocolate: Uma revisão. Curso de Tecnologia de Alimentos da Faculdade de Tecnologia de Marília, São Paulo, 14 p., 2010.

KWIKURIBE, C. Potential Health Benefits of Cocoa Flavanols. The Manufacturing Confectioner, Princeton, v. 85, n. 10, p. 4349, 2005.

LIPPI, G., FRANCHINI, M., MONTAGNANA, M., FAVALORO, E. J., GUIDI, G. C., TARGHER, G. D. Chocolate: consumption for pleasure or therapy? Journal of Thrombosis and Thrombolysis , v.28, n.4, p.482488, 2009.

LORENZO, N. D. Mesocarpo do pequi (Caryocar villosum Alb. Pers.): incorporação em formulação de chocolate amargo com vista a agregação de valor nutricional. São Paulo, 2017. 124p

OLIVEIRA, L. P. M.; MORAES, C. M. B.; SILVA, G. L.; OLIVEIRA, J. R. Comparação da atividade antioxidante in vitro do cacau (Theobroma Cacao L.) fermentado e não fermentado. RESUMO DO X SALÃO DE INICIAÇÃO CIENTÍFICA PUCRS, p. 5355. 2009.

PIMENTEL, F. A. Avaliação do poder antioxidante do chocolate amargo: um comparativo com o vinho tinto. Dissertação (Mestrado em Ciência e Tecnologia de Alimentos). Universidade Federal do Rio Grande do Sul. 82 p. Porto Alegre, Fevereiro, 2007.

RICHTER, M; LANNES, S.C.S. Ingredientes usados na indústria de chocolates. Revista Brasileira de Ciências Farmacêuticas, v. 43, n. 3, São Paulo, 2007. 
SOUZA, A.S.L. Avaliação da estabilidade térmica e oxidativa de chocolates amargos. 2010. 110f. Dissertação (Pós Graduação em Ciência e Tecnologia de Alimentos) Universidade Federal da Paraíba, João Pessoa, 2010.

UNIVERSIDADE ESTADUAL DE CAMPINAS - UNICAMP. Tabela brasileira de composição de alimentos - TACO. 4. ed. rev. e ampl. Campinas: UNICAMP / NEPA, 2011. 161 p. Disponível em: <http://www.unicamp.br/nepa/taco/tabela. php?ativo=tabela $>$. Acesso em: 19 jun. 2018.

VISSOTTO, F.Z. LUCCAS, V. BRAGAGNOLO, N. TURATTI, J.M. GRIMALDI, R. FIGUEIREDO, M.S. de. Caracterização Físico-química e reológica de chocolates comerciais tipo cobertura e elaborados com gorduras alternativas. Brazilian Journal of Food technology, Campinas, v.2, p.139-148, 1999. 